\title{
High Prevalence of Hearing Impairment in Primary Congenital Hypothyroidism
}

\author{
Tal Almagor $^{a, b, c}$ Shoshana Rath ${ }^{a}$ Dan Nachtigal ${ }^{d}$ Zohara Sharroni $^{d}$ \\ Ghadir Elias-Assad $^{\mathrm{a}, \mathrm{c}}$ Ora Hess ${ }^{\mathrm{a}}$ Gilad Havazelet $^{\mathrm{e}}$ Yoav Zehavi $^{\mathrm{b}}$ \\ Ronen Spiegel ${ }^{b, c}$ Dani Bercovich ${ }^{f, g}$ Shlomo Almashanu ${ }^{\text {h }}$ \\ Yardena Tenenbaum-Rakovera, c
}

aPediatric Endocrine Institute, Ha'Emek Medical Center, Afula, Israel; bPediatric Department B, Ha'Emek Medical Center, Afula, Israel; 'Rappaport Faculty of Medicine, Technion - Israel Institute of Technology, Haifa, Israel;

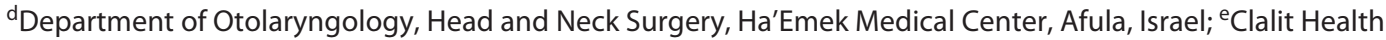
Services, North District, Israel; ${ }^{f}$ Faculty of Medical Science, Tel Hai Academic College Upper Galilee, Tel Hai, Israel; gGGA - Galil Genetic Analysis Laboratory Ltd., Kazerin, Israel; ${ }^{\text {h} T h e ~ N a t i o n a l ~ N e w b o r n ~ S c r e e n i n g ~ P r o g r a m, ~ M i n i s t r y ~ o f ~}$ Health, Tel-HaShomer, Israel

\section{Keywords}

Primary congenital hypothyroidism · Deafness .

Hearing impairment . Conductive hearing impairment .

Sensorineural hearing impairment

\begin{abstract}
Background: An association between hearing impairment $(\mathrm{HI})$ and congenital hypothyroidism $(\mathrm{CH})$ has been reported previously. However, in general, studies were retrospective and had small sample sizes, and the results were variable and inconclusive. The aim of our study was to assess the prevalence of $\mathrm{HI}$ among patients with $\mathrm{CH}$ and to examine factors potentially predictive of $\mathrm{HI}$ including severity of $\mathrm{CH}$, etiology of $\mathrm{CH}$, and timing of treatment initiation. Methods: Audiometry was undertaken prospectively in 66 patients aged 3-21 years diagnosed with primary $\mathrm{CH}$ and 49 healthy matched controls. All patients with $\mathrm{HI}$ underwent examination by an otolaryngologist, and in patients with sensorineural loss, brainstem evoked response audiometry was performed. A next-generation sequencing (NGS) panel for genes involved in deafness was performed in patients with sensorineural $\mathrm{HI}$ to exclude additional genetic etiologies. Results: HI was
\end{abstract}

found in 19 patients (28.7\%). Among them, 5 (7.6\%) had moderate to severe bilateral sensorineural impairment and $14(21.2 \%)$ had mild conductive HI. Conductive HI was bilateral in 5 of these patients (36\%). None of the controls had HI. No specific etiology was found in patients with $\mathrm{HI}$, and no differences were identified in age at diagnosis, age at initiation of levothyroxine $\left(\mathrm{LT}_{4}\right)$ therapy, gender, or ethnicity between patients with and without $\mathrm{HI}$. A nonsignificant trend toward lower mean screening $\Pi_{4}$ levels was found in patients with $\mathrm{HI}$ (compared to those without $\mathrm{HI}$ ) (3.42 vs. 5.34 $\mu \mathrm{g} / \mathrm{dL}, p=0.095)$. No pathogenic variants in genes attributed to $\mathrm{HI}$ were identified by NGS in the 5 patients with sensorineural deafness, indicating that $\mathrm{HI}$ in these patients was likely attributable to $\mathrm{CH}$ rather than other genetic etiologies. Conclusions: Our findings indicate a high prevalence of $\mathrm{HI}$ among patients with $\mathrm{CH}$, predominantly of the conductive type. $\mathrm{HI}$ was not associated with the etiology of $\mathrm{CH}$ or with delayed initiation of $\mathrm{LT}_{4}$ therapy. Audiometry is recommended for children diagnosed with $\mathrm{CH}$ and repeat monitoring may be warranted to identify acquired $\mathrm{HI}$ and to prevent long-term sequelae of undiagnosed deafness.

(C) 2020 European Thyroid Association Published by S. Karger AG, Basel

\section{karger@karger.com \\ www.karger.com/etj \\ (C) 2020 European Thyroid Association \\ Published by S. Karger AG, Basel}

Karger!
Yardena Tenenbaum-Rakover

Pediatric Endocrine Institute, Ha'Emek Medical Center

24 Yitzhak Rabin Ave.

Afula 1834111 (Israel)

rakover_y@clalit.org.il 


\section{Introduction}

The development of hearing in humans, as well as in rodents, is highly dependent on a sufficient supply of thyroid hormones (TH). TH deprivation during the late fetal period, or delay in TH replacement therapy in infancy may result in hearing impairment (HI) [1-3]. Deaf-mutism and $\mathrm{HI}$ are common in areas of iodine deficiency [4] and were reported in patients with congenital hypothyroidism $(\mathrm{CH})$ prior to the implementation of thyroid neonatal screening in the 1980s [5].

TH play an important role in the development of the inner ear and auditory pathway [6-13]. In animal models, inadequate TH supply before inner ear development results in permanent cochlear defects [3, 7]. Malformations of the organ of Corti have been shown in offspring of female mice with chemically induced hypothyroidism [8]. Moreover, TH influence the development and maturation of the middle ear and the size of the ossicular bones. This association has been demonstrated in mice lacking TH receptors Thra and $\operatorname{Thr} \beta$ [12].

In humans, few studies have investigated $\mathrm{HI}$ in patients with $\mathrm{CH}$; however, the samples were small, and the studies were generally retrospective with wide variable results [1418]. A nationwide study in France of 1,202 young adult patients diagnosed with $\mathrm{CH}$ and treated early reported a 3 -fold risk of hearing loss compared to the normal population [14]. Other studies with smaller sample sizes indicated $\mathrm{HI}$ prevalence of $20-47 \%$ in patients with $\mathrm{CH}$, primarily sensorineural in nature [14-16]. HI was associated with the severity of $\mathrm{CH}$ and with prenatal onset of hypothyroidism [18] and the age of therapy initiation [17]. In contrast, other studies have demonstrated low rates of $\mathrm{HI}$ in patients with $\mathrm{CH}$ [19-22]. The objective of this study was to assess the prevalence of $\mathrm{HI}$ among patients with $\mathrm{CH}$ and to evaluate whether the severity of $\mathrm{CH}$, the etiology, early initiation of treatment, or other factors predispose to HI.

\section{Materials and Methods}

\section{Subjects}

Sixty-six patients aged 3.1-21.9 years (mean age 8.65) were prospectively recruited from a cohort of 150 patients diagnosed with primary $\mathrm{CH}$. All 66 patients included in the study agreed to participate and underwent audiometry testing. All were followed at the Pediatric Endocrinology Institute, Ha'Emek Medical Center. The diagnosis of $\mathrm{CH}$ was based on abnormal thyroid function at birth; most patients were identified by the National Thyroid Newborn Screening Program. The etiology of the $\mathrm{CH}$ was evaluated by ${ }^{99} \mathrm{Tc}$ scan, performed at the age of $2-3$ years after 3 weeks off levothyroxine $\left(\mathrm{LT}_{4}\right)$ therapy. Patients were followed every 3 months in the first year and 6 monthly thereafter. $\mathrm{LT}_{4}$ was adjusted to maintain TSH within the normal range. Patients with a history of recurrent otitis media, Pendred syndrome, or other known syndromes such as Down syndrome were excluded from the study.

In cases with familial $\mathrm{CH}$, the candidate gene approach was applied to identify the molecular genetic etiology of $\mathrm{CH}$. A control group of 49 healthy children aged 4.8-17.7 years (mean age 8.98), matched for age, sex, and ethnicity was recruited. Exclusion criteria for the control group included patients with known tympanic perforations, recurrent otitis media, developmental delay, congenital syndromes, intrauterine infections, and prematurity. Clinical and biochemical data were retrieved from the participants' medical files. The biochemical parameters included TSH and total (T) $\mathrm{T}_{4}$ levels on neonatal screening, and laboratory TSH and free (F) $\mathrm{T}_{4}$ levels measured prior to initiation of $\mathrm{LT}_{4}$ therapy.

\section{National Neonatal Screening}

Blood samples were collected by heel puncture $48-72 \mathrm{~h}$ after birth. Between 1987 and 2006, the Israeli National Newborn Screening Laboratory performed the tests using a Diagnostic Products Corp. (Los Angeles, CA, USA) radioimmunoassay $\mathrm{TT}_{4}$ and TSH kits. Since 2006, Perkin Elmer B065-112 AutoDELFIA neonatal $\mathrm{TT}_{4}$ and B032-312 AutoDELFIA neonatal TSH kits have been employed, both of which utilize time-resolved fluoroimmunoassays (PerkinElmer Life and Analytical Sciences, Wallac Oy, Mustionkatu 6, Turku, Finland). The Israeli $\mathrm{CH}$ screening program is based on $\mathrm{TT}_{4}$ level, followed by confirmatory TSH test, such that when the level of $\mathrm{TT}_{4}$ is below the 10th percentile for age, TSH is measured. TSH values $>20 \mathrm{mIU} / \mathrm{L}$ are considered indicative of primary $\mathrm{CH}$. Neonates with abnormal screening results are referred to medical centers. The results of screening TSH were not reported in accurate values but rather as $>20 \mathrm{mIU} / \mathrm{L}$; therefore, we could not calculate precisely the screening TSH results.

\section{Hormone Analyses}

$\mathrm{TSH}, \mathrm{FT}_{4}$, and $\mathrm{FT}_{3}$ were measured by direct automated chemiluminescent immunoradiometric assay using the ADVIA Centaur immunoassay system (Bayer Corporation, Tarrytown, NY, USA). Laboratory TSH reference values were $0.4-4.2 \mathrm{mIU} / \mathrm{L}$, and $\mathrm{FT}_{4}$ normal reference was $10-20 \mathrm{nmol} / \mathrm{L}$. Some of the TSH values were above the higher standard of the method. In these cases, no dilutions were performed, precluding calculation of the precise TSH values. TSH results were therefore not presented.

\section{Audiometry Test}

Patients or their parents if they were below 18 years of age signed an appropriate informed consent form for audiometry and underwent conventional pure tone audiometry at a single institute using GSI AudioStar Pro ${ }^{\mathrm{TM}}$ Grason Stadler audiometer. HI was classified as sensorineural or conductive. Mild HI was defined as threshold hearing between 21 and $40 \mathrm{~dB}$, moderate $\mathrm{HI}$ between 41 and $70 \mathrm{~dB}$, and severe $\mathrm{HI}$ above $70 \mathrm{~dB}$. HI was referred as bilateral or unilateral and of low frequency when the HI was below $1,000 \mathrm{~Hz}$ and of high frequency when it was above $2,000 \mathrm{~Hz}$. The same otolaryngologist evaluated the results of all audiometry tests. Patients with HI underwent otolaryngology evaluation including otoscopy, and in patients with sensorineural loss, brainstem evoked response audiometry. The study was approved by the institute's Ethics Committee and by the Israeli Ministry of Health. 
Table 1. Clinical and biochemical characteristics of the study group and of patients with $\mathrm{HI}$

\begin{tabular}{lcc}
\hline & All patients & Patients with HI \\
\hline Patients, $n$ & 66 & 19 \\
Age, years & $8.65 \pm 5.1(3.1-21.9)$ & $10.88 \pm 6.4(3.1-21.9)$ \\
Gender $(\mathrm{M}: \mathrm{F})$ & $34: 32$ & $10: 9$ \\
Age at diagnosis of CH, days & $11.08 \pm 9.0(2-49)$ & $11.19 \pm 7.0(2-24)$ \\
Age at initiation of $\mathrm{LT}_{4}$ therapy, days & $13.21 \pm 10.8(2-62)$ & $13.27 \pm 6.3(2-24)$ \\
Screening $\mathrm{TT}_{4}, \mu \mathrm{g} / \mathrm{dL}$ & $4.7 \pm 2.9(0.5-13)$ & $3.4 \pm 2.0(0.8-8.2)$ \\
Laboratory FT, $\mathrm{Fmol}_{4}, \mathrm{~L}$ & $5.6 \pm 4.6(0.3-19.2)$ & $4.03 \pm 2.4(0.3-8)$ \\
\hline Etiology & & 9 \\
Dysgenesis & $41(62 \%)$ & 2 \\
$\quad$ Agenesis & 13 & 7 \\
Ectopic thyroid & 28 & 7 \\
Dyshormonogenesis & $19(29 \%)$ & 0 \\
TPO mutations & 12 & 0 \\
TSHR mutations & 6 & 2 \\
Thyroglobulin mutation & 1 & 0 \\
Transient CH & $5(7.6 \%)$ & \\
Unknown & $1(1.4 \%)$ & \\
\hline
\end{tabular}

Values denote mean $\pm \mathrm{SD}$ (range) or $n(\%)$. HI, hearing impairment; $\mathrm{CH}$, congenital hypothyroidism; $\mathrm{LT}_{4}$, levothyroxine; $\mathrm{TT}_{4}$, total $\mathrm{T}_{4} ; \mathrm{FT}_{4}$, free $\mathrm{T}_{4} ; \mathrm{TPO}$, thyroid peroxidase; TSHR, $\mathrm{TSH}$ receptor.

\section{Genetic Analysis}

In patients diagnosed with thyroid dyshormonogenesis, a candidate gene approach was performed to identify the etiology of the $\mathrm{CH}$. In cases with a known familial mutation, a targeted gene-sequencing approach was employed in the proband.

In view of the high rate of consanguinity in our population, next-generation sequencing (NGS) was performed in patients with sensorineural HI using a hearing loss panel to exclude other genetic etiologies for hearing loss. The panel included 198 genes associated with deafness. NGS was performed by Illumina NextSeq and $97 \%$ of the variants were identified by BWA algorithm and analyzed using variant studio (Illumina), ANNOVAR, and Genoox software (see online suppl. material 1; for all online suppl. material, see www.karger.com/doi/10.1159/000509775).

\section{Statistical Methods}

Statistical analyses were performed using the SAS software package version 9.4 (SAS Institute, Cary, NC, USA). A series of $\chi^{2}$ tests or Fisher's exact tests (when the assumptions of the parametric $\chi^{2}$ test were not met) and a nonparametric Mann-Whitney $U$ test were conducted to analyze the difference between patients' characteristics in both groups. We computed the 2-tailed $p$ values, where $p<0.05$ was considered statistically significant.

\section{Results}

Characteristics of the patients diagnosed with $\mathrm{CH}$ are given in Table 1. CH was caused by thyroid dysgenesis in $62 \%$ (agenesis or ectopic thyroid), dyshormonogenesis in
29\% (thyroid peroxidase [TPO], TSH receptor [TSHR], or thyroglobulin gene mutations), and transient $\mathrm{CH}$ in $7.6 \%$ of patients (Table 1). Maternal thyroid disorders were not reported for any patients included in the study. The control group included 49 patients with a mean age of 8.98 years. No significant differences were found between the controls and the study group in gender, ethnicity, or age at the time of the study.

HI was identified in $29 \%$ of the $\mathrm{CH}$ patients (Table 2), whereas all controls had normal hearing. Among patients with HI, 14 (74\%) had conductive hearing loss and 5 (26\%) had sensorineural hearing loss. Conductive HI was mild in all patients and found to be bilateral in $5(36 \%)$ at variable frequencies (44\% with high frequency and 56\% with low frequency). All 5 patients with sensorineural hearing loss had moderate to severe bilateral impairment. Otoscope examination in all HI patients was normal, except for 1 patient who had mild serous otitis media. A comparison between patients with and without HI revealed no differences in age at diagnosis, age at $\mathrm{LT}_{4}$ initiation, gender, ethnicity, or $\mathrm{CH}$ etiology (Table 2). A nonsignificant trend toward lower screening $\mathrm{TT}_{4}$ was noted in patients with hearing loss $(3.42 \mathrm{vs} .5 .34 \mu \mathrm{g} / \mathrm{dL}$, $p=0.095$ ) (Table 2). The etiology of $\mathrm{CH}$ was variable among patients with $\mathrm{HI}$ : those with conductive hearing loss had ectopic thyroid gland (6 patients), TPO mutation 
Table 2. Characteristics of patients with and without HI

\begin{tabular}{lccc}
\hline & Normal hearing & HI & $p$ value \\
\hline Patients, $n$ & 47 & 19 & 0.153 \\
Age at the study, years & $7.7 \pm 4.25(3.1-17.4)$ & $10.98 \pm 6.4(3.1-21.9)$ & 0.566 \\
Age at diagnosis, days & $11.0 \pm 9.94(3.0-49.0)$ & $11.2 \pm 7.0(2-24)$ & 0.245 \\
Age at $\mathrm{LT}_{4}$ initiation, days & $13.2 \pm 12.55(3.0-62.0)$ & $13.26 \pm 6.3(2-24)$ & 0.909 \\
Gender $(\mathrm{M}: \mathrm{F})$ & $24 / 23$ & $10 / 9$ & 0.095 \\
Screening $\mathrm{TT}_{4}, \mu \mathrm{\mu g} / \mathrm{dL}$ & $5.3 \pm 3.06(0.5-13.0)$ & $3.4 \pm 2.0(0.8-8.2)$ & 0.450 \\
Laboratory $\mathrm{FT}_{4}, \mathrm{pmol} / \mathrm{L}$ & $6.1 \pm 5.0(0.4-19.2)$ & $4.0 \pm 2.4(0.3-8.0)$ & \\
\hline
\end{tabular}

Values denote mean $\pm \mathrm{SD}$ (range) or $n(\%)$. HI, hearing impairment; $\mathrm{LT}_{4}$, levothyroxine; $\mathrm{TT}_{4}$, total $\mathrm{T}_{4} ; \mathrm{FT}_{4}$, free $\mathrm{T}_{4}$.

Table 3. Clinical and laboratory characteristics of 5 patients with sensorineural HI

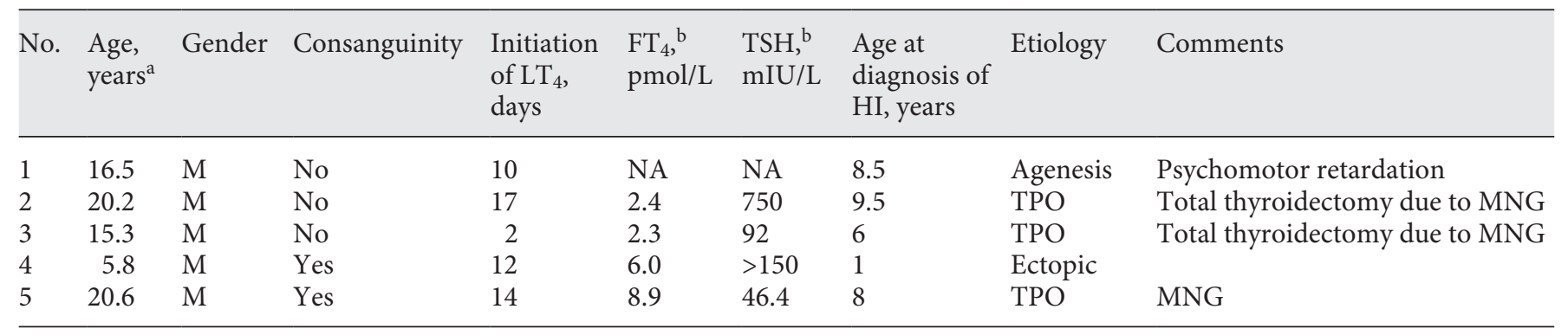

Patients 2 and 3 are brothers. HI, hearing impairment; $\mathrm{LT}_{4}$, levothyroxine; $\mathrm{FT}_{4}$, free $\mathrm{T}_{4}$; $\mathrm{TPO}$, thyroid peroxidase; MNG, multinodular goiter. ${ }^{a}$ Age at time of study. ${ }^{b}$ At diagnosis.

(4 patients), thyroid agenesis (1 patient), TSHR mutation (1 patient), or transient $\mathrm{CH}$ (2 patients), and those with sensorineural HI had TPO mutation, ectopic sublingual thyroid, or thyroid agenesis (Table 3). Detailed characteristics of NGS sequencing performed on the 5 patients with sensorineural $\mathrm{HI}$ are presented in Table 4. Although 4 variants in the heterozygous state were identified in 3 patients, they were not associated with clinical phenotypes (online suppl. material 2).

\section{Discussion}

In this prospective study, we identified $\mathrm{HI}$ in almost $30 \%$ of patients with $\mathrm{CH}$, among them $74 \%$ with mild conductive impairment and $26 \%$ with moderate to severe sensorineural impairment. HI was not associated with the etiology of $\mathrm{CH}$, gender, ethnicity, and the age at therapy initiation. A nonsignificant trend toward lower screening $\mathrm{TT}_{4}$ was observed. No previous study has conclusively excluded other genetic etiologies for $\mathrm{HI}$ in patients with $\mathrm{CH}$. We utilized NGS panel testing to exclude other genetic causes, confirming that sensorineural HI is likely attributable to $\mathrm{CH}$.

The prevalence of HI in the USA is 1.6:1,000 newborns [23] and $4 \%$ in people younger than 45 years [24]. In Israel, since 2010, all newborns undergo hearing screening using transient evoked otoacoustic emission (TEOAE) at 2 days of age. Those that do not pass are referred for brainstem evoked response audiometry. The precise prevalence of $\mathrm{HI}$ in the Israeli population is unknown, but $0.5 \%$ of newborns in Israel do not pass the transient evoked otoacoustic emission screening [25]. Only 4 patients in our cohort were born after 2010 and none had HI detected on screening. The lack of newborn audiometry screening in most of our cohort might explain the delay in the diagnosis of HI among our patients. However, it is likely that some hearing loss in $\mathrm{CH}$ develops over time and is not necessarily congenital, such that repeated screening is necessary. In this study, we found a high prevalence of $\mathrm{HI}$ in $\mathrm{CH}$ patients, whereas control patients were unaffected. HI early in life results in delayed language acquisition and has an impact on learning and cognitive abilities [26,27]. A previous study based on self- 
Table 4. Audiometry results and genetic findings using next-generation sequencing in 5 patients with sensorineural hearing impairment

\begin{tabular}{lllllll}
\hline No. & Audiometry result & & NGS panel & \multicolumn{2}{l}{ Variant } \\
\hline 1 & Bilateral & Mild-moderate & High frequency & Hetero-PCDH15 & c.4603_4607delCAAGT, p.Gln535ILefs 25 & VUS \\
\cline { 3 - 6 } & & & Hetero-SFS1 & c.2129C>G; p.Thr710Ser & rs.200136995 \\
\hline 2 & Bilateral & Moderate-severe & All frequencies & No pathological variant & \\
\hline 3 & Bilateral & Moderate & All frequencies & Hetero-CACNA1D & c.298T>C; p.Ser97Pro & VUS \\
\hline 4 & Bilateral & Left mild & High frequency & No pathological variant & \\
\hline 5 & Bilateral & Moderate-severe & All frequencies & Hetero-COL4A3 & c.3321_3329delAAGTCCTGG; p.Ser1108_Gly1110del VUS \\
\hline
\end{tabular}

Patients 2 and 3 are brothers diagnosed with homozygous TPO mutation (c.1618C >T; p.R540X), patient 5 has homozygous TPO mutation (c.875C $>\mathrm{T}$; S292F), both previously described [35]. NGS, next-generation sequencing; Hetero, heterozygous; VUS, variant of uncertain significance; TPO, thyroid peroxidase.

reported data indicated a 3 -fold higher rate of $\mathrm{HI}$ (predominantly sensorineural) among $\mathrm{CH}$ patients compared to the normal population, with a particularly high incidence in patients with thyroid agenesis and severe disease at diagnosis (as assessed by delayed epiphyseal ossification) [14]. In other studies, HI was associated with the severity of $\mathrm{CH}[16,18]$, age of therapy initiation [17], and prenatal onset hypothyroidism [18] but not with the etiology of $\mathrm{CH}$ [16]. In our study, a trend toward lower neonatal screening $\mathrm{TT}_{4}$ was identified in patients with $\mathrm{HI}$, supporting an association with more severe $\mathrm{CH}$ at diagnosis. No correlation was found between $\mathrm{HI}$ and various etiologies of $\mathrm{CH}$, nor was the prevalence higher in infants with thyroid agenesis.

Delayed initiation of $\mathrm{LT}_{4}$ supplemental therapy or inadequate therapy results in delayed psychomotor development and is associated with higher rates of HI [2]. Interestingly, in our study, there was no difference in the age at initiation of supplemental therapy between patients with $\mathrm{HI}$ and those with normal hearing. Mean age at $\mathrm{LT}_{4}$ initiation was 13.21 days but age ranged from 2 to 62 days. All patients were diagnosed after the implementation of neonatal screening in Israel; nonetheless, diagnosis was delayed in some. Patients born in the last decade were diagnosed earlier and $\mathrm{LT}_{4}$ was initiated sooner than in older patients. Moreover, the $\mathrm{LT}_{4}$ dose at initiation of therapy has increased from 7 to $10-15 \mu \mathrm{g} /$ day in the last decade. These changes in the management of infants with $\mathrm{CH}$ may have impacted the study outcome.

TH play a key role in auditory system development. Studies in rodents have identified the cochlea as a major site of TH action. Prenatal TH deficiency causes impaired maturation of the sensory epithelium and delayed myelination of the cochlear nerve, resulting in permanent deafness [11] and TH supplement administered during critical period of hearing development can prevent deafness in CH mice [28]. THR $\beta$ is expressed in the inner ears, and mice lacking Thr have cochlear deafness [29].

Prior to the widespread implementation of neonatal screening in the 1980s, children with $\mathrm{CH}$, as well as those with endemic cretinism due to iodine deficiency, presented with clinical phenotypes of severe cognitive and neurological impairment, extreme short stature [4], hearing loss, and deaf-mutism [4, 5]. About $20 \%$ of patients with $T H R \beta$ mutations exhibit hearing loss, including conductive $\mathrm{HI}$ [30], and about 50\% have recurrent ear infections [31].

In our study, a higher rate of conductive HI was identified than in previous studies, in which sensorineural HI predominated [14-17]. As already noted, TH receptor controls maturation of the middle ear and the size of the ossicular bones in animal models [12]. Patients with THR $\beta$ mutations exhibit HI, including the conductive type. Furthermore, conductive $\mathrm{HI}$ has been shown to be associated with nonadherence to thyroid supplement therapy in patients with acquired hypothyroidism [32]. All these suggest that $\mathrm{TH}$ have a role in hearing beyond the neonatal period. In our study, the diagnosis of HI occurred at a mean age of 8.65 years, but the age of onset is unclear, as is the natural history. Conductive losses can fluctuate, deteriorate, or improve with age. Furthermore, adherence to therapy may also impact. The prevalence of sensorineural and conductive deficits identified in our cohort highlights the importance of neonatal screening, as well as regular 
screening throughout childhood for children with $\mathrm{CH}$, to facilitate early intervention and minimize the developmental impact of $\mathrm{HI}$ in this high-risk population.

In patients found to have sensorineural deafness, we performed an NGS panel including the known genes associated with $\mathrm{HI}$, in order to exclude other genetic causes for HI. We identified a high prevalence of variants in genes reportedly associated with $\mathrm{HI}$; however, all variants were heterozygous and patients did not present with phenotypes described with homozygous mutations in these genes. However, we cannot exclude that heterozygous variants may act as modulating factors, exacerbating the severity of $\mathrm{HI}$ in patients with $\mathrm{CH}$ or increasing the risk of $\mathrm{HI}$ in the setting of hypothyroidism. Interestingly, 3 of the 5 patients with sensorineural HI had TPO mutations. TPO mutations in association with sensorineural deafness have been reported previously in some case reports [33-35], as well as in animal models [36], indicating that the TPO enzyme itself may have a role in the development of the auditory system.

This prospective, controlled study benefited from having all audiometry performed at a single center and interpreted by 1 physician, ensuring consistent results. In addition, we were able to exclude other genetic etiologies of HI by using the recently developed NGS panel for genetic etiologies of congenital deafness. The study is limited by its relatively small number of participants, which may have obscured an association between $\mathrm{HI}$ and initial severity or etiology of $\mathrm{CH}$. Further studies are required to determine the age at development of $\mathrm{HI}$ and to investigate long-term outcomes.

\section{Conclusions}

HI was identified in nearly $30 \%$ of patients with $\mathrm{CH}$ and was predominantly conductive in nature, affecting both high and low frequencies. Twenty-six percent had moderate to severe sensorineural loss, and in all of them, other genetic causes of sensorineural HI were excluded.
HI was not associated with the etiology of $\mathrm{CH}$ or with delayed initiation of $\mathrm{LT}_{4}$ therapy. Audiometry is recommended for children diagnosed with $\mathrm{CH}$, and repeat monitoring may be warranted to identify acquired $\mathrm{HI}$ and to prevent long-term sequelae of undiagnosed deafness.

\section{Acknowledgements}

We thank Ayellet Jehassi for the statistical analysis and Camille Vainstein for the professional English editing.

\section{Statement of Ethics}

The study protocol was approved by the institute's committee of Ha'Emek Medical Center and the Israeli Ministry of Health (HT 5226).

\section{Conflict of Interest Statement}

All authors have nothing to disclose.

\section{Funding Sources}

This research was supported by a grant from the Academic Committee of Ha'Emek Medical Center, Afula, Israel.

\section{Author Contributions}

T.A. designed the study, collected and evaluated the data, drafted the initial manuscript, and reviewed and revised the manuscript; Y.T.-R. designed the study, collected and evaluated the data, and reviewed carefully and revised the manuscript; D.N. and Z.S. performed and interpreted the audiometry tests and reviewed the manuscript; S.R., G.E.-A., O.H., and G.H. collected data and reviewed and revised the manuscript; Y.Z., R.S., and D.B. interpreted the molecular genetic results and reviewed and revised the manuscript; S.A. collected thyroid screening data and reviewed the manuscript. All authors approved the final manuscript as submitted and agree to be accountable for all aspects of the work.

\section{References}

1 Sohmer H, Freeman S. The importance of thyroid hormone for auditory development in the fetus and neonate. Audiol Neurootol. 1996;1(3):137-47.

2 Andrade CLO, Machado GC, Fernandes LDC, Albuquerque JM, Casais-E-Silva LL, Ramos $\mathrm{HE}$, et al. Mechanisms involved in hearing disorders of thyroid ontogeny: a literature review. Arch Endocrinol Metab. 2017;61(5):501-5.
3 Heuer H. Hear, hear! Thyroid hormone transporters in cochlear development. Endocrinology. 2011;152(12):4478-80.

4 DeLong GR, Stanbury JB, Fierro-Benitez R. Neurological signs in congenital iodine-deficiency disorder (endemic cretinism). Dev Med Child Neurol. 2008;27(3):317-24.
5 Hulse A. Congenital hypothyroidism and neurological development. J Child Psychol Psychiatry. 1983;24(4):629-35.

6 Knipper M, Zinn C, Maier H, Praetorius M, Rohbock K, Köpschall I, et al. Thyroid hormone deficiency before the onset of hearing causes irreversible damage to peripheral and central auditory systems. J Neurophysiol. 2000;83(5):3101-12. 
7 O’Malley BW, Li D, Turner DS. Hearing loss and cochlear abnormalities in the congenital hypothyroid (hyt/hyt) mouse. Hear Res. 1995; 88:181-9.

8 Deol MS. The role of thyroxine in the differentiation of the organ of Corti. Acta Otolaryngol. 1976;81(5-6):429-35.

9 Uziel A, Pujol R, Legrand C, Legrand J. Cochlear synaptogenesis in the hypothyroid rat. Brain Res. 1983;283(2-3):295-301.

10 Berbel P, Guadaño-Ferraz A, Martínez M, Quiles JA, Balboa R, Innocenti GM. Organization of auditory callosal connections in hypothyroid adult rats. Eur J Neurosci. 1993; 5(11):1465-78.

11 Sharlin DS, Visser TJ, Forrest D. Developmental and cell-specific expression of thyroid hormone transporters in the mouse cochlea Endocrinology. 2011;152(12):5053-64.

12 Cordas EA, Ng L, Hernandez A, Kaneshige M, Cheng SY, Forrest D. Thyroid hormone receptors control developmental maturation of the middle ear and the size of the ossicular bones. Endocrinology. 2011;153(3):1548-60.

13 Ng L, Goodyear RJ, Woods CA, Schneider MJ, Diamond E, Richardson GP, et al. Hearing loss and retarded cochlear development in mice lacking type 2 iodothyronine deiodinase. Proc Natl Acad Sci U S A. 2004;101(10): 3474-9.

14 Lichtenberger-Geslin L, Dos Santos S, Hassani Y, Ecosse E, Van Den Abbeele T, Léger J. Factors associated with hearing impairment in patients with congenital hypothyroidism treated since the neonatal period: a national population-based study. J Clin Endocrinol Metab. 2013;98(9):3644-52.

15 Rovet J, Walker W, Bliss B, Buchanan L, Ehrlich R. Long-term sequelae of hearing impairment in congenital hypothyroidism. J Pediatr. 1996;128(6):776-83.

16 Vanderschueren-Lodeweyckx M, Debruyne F, Dooms L, Eggermont E, Eeckels R. Sensorineural hearing loss in sporadic congenital hypothyroidism. Arch Dis Child. 1983;58(6): $419-22$.
17 Wasniewska M, De Luca F, Siclari S, Salzano G, Messina MF, Lombardo F, et al. Hearing loss in congenital hypothalamic hypothyroidism: a wide therapeutic window. Hear Res. 2002;172(1-2):87-91.

18 Bruno R, Aversa T, Catena M, Valenzise M, Lombardo F, De Luca F, et al. Even in the era of congenital hypothyroidism screening mild and subclinical sensorineural hearing loss remains a relatively common complication of severe congenital hypothyroidism. Hear Res. 2015;327:43-7.

19 Bellman SC, Davies A, Fuggle PW, Grant DB, Smith I. Mild impairment of neuro-otological function in early treated congenital hypothyroidism. Arch Dis Child. 1996;74(3):215-8.

20 François M, Bonfils P, Leger J, Czernichow P, Narcy P. Role of congenital hypothyroidism in hearing loss in children. J Pediatr. 1994; 124(3):444-6

21 Hashemipour M, Hovsepian S, Hashemi M, Amini M, Kelishadi R, Sadeghi S. Hearing impairment in congenitally hypothyroid patients. Iran J Pediatr. 2012;22(1):92-6.

22 Dayal D, Hansdak N, Vir D, Gupta A, Bakshi J. Hearing impairment in children with permanent congenital hypothyroidism: data from Northwest India. Thyroid Res Pract. 2016;13:67-70.

23 Centers for Disease Control and Prevention (CDC). Identifying infants with hearing loss: United States, 1999-2007. MMWR Morb Mortal Wkly Rep. 2010;59(8):220-3.

24 Nadol JB Jr. Hearing loss. N Engl J Med. 1993; 329(15):1092-102.

25 Attias J, Al-Masri M, Abukader L, Cohen G, Merlov P, Pratt H, et al. The prevalence of congenital and early-onset hearing loss in Jordanian and Israeli infants. Int J Audiol. 2006; 45(9):528-36.

26 Conway CM, Pisoni DB, Kronenberger WG. The importance of sound for cognitive sequencing abilities: the auditory scaffolding hypothesis. Curr Dir Psychol Sci. 2009;18(5): 275-9.

27 Davis JM, Elfenbein J, Schum R, Bentler RA. Effects of mild and moderate hearing impairments on language, educational, and psychosocial behavior of children. J Speech Hear Disord. 1986;51(1):53-62.
28 Karolyi IJ, Dootz GA, Halsey K, Beyer L, Probst FJ, Johnson KR, et al. Dietary thyroid hormone replacement ameliorates hearing deficits in hypothyroid mice. Mamm Genome. 2007;18(8):596-608.

29 Forrest D, Erway LC, Ng L, Altschuler R, Curran $\mathrm{T}$. Thyroid hormone receptor beta is essential for development of auditory function. Nat Genet. 1996;13(3):354-7.

30 Brucker-Davis F, Skarulis MC, Pikus A, Ishizawar D, Mastroianni MA, Koby M, et al. Prevalence and mechanisms of hearing loss in patients with resistance to thyroid hormone. J Clin Endocrinol Metab. 1996;81(8):276872.

31 Brucker-Davis F, Skarulis MC, Grace MB, Benichou J, Hauser P, Wiggs E, et al. Genetic and clinical features of 42 kindreds with resistance to thyroid hormone. The National Institutes of Health Prospective Study. Ann Intern Med. 1995;123(8):572-83.

32 Vikas M, Shukla G, Naresh B. Hearing profile in hypothyroidism. Indian J Otolaryngol Head Neck Surg. 2002;54:285-90.

33 Pfarr N, Borck G, Turk A, Napiontek U, Keilmann A, Müller-Forell W, et al. Goitrous congenital hypothyroidism and hearing impairment associated with mutations in the TPO and SLC26A4/PDS genes. J Clin Endocrinol Metab. 2006;91(7):2678-81.

34 Chertok Shacham E, Ishay A, Irit E, Pohlenz J, Tenenbaum-Rakover Y. Minimally invasive follicular thyroid carcinoma developed in dyshormonogenetic multinodular goiter due to thyroid peroxidase gene mutation. Thyroid. 2012;22(5):542-6.

35 Tenenbaum-Rakover Y, Mamanasiri S, RisStalpers C, German A, Sack J, Allon-Shalev S, et al. Clinical and genetic characteristics of congenital hypothyroidism due to mutations in the thyroid peroxidase (TPO) gene in Israelis. Clin Endocrinol. 2007;66(5):695-702.

36 Johnson KR, Gagnon LH, Longo-Guess CM, Harris BS, Chang B. Hearing impairment in hypothyroid dwarf mice caused by mutations of the thyroid peroxidase gene. J Assoc Res Otolaryngol. 2014;15(1):45-55. 